\title{
PURPOSE AND BENEFITS OF HYBRID SIMULATION: CONTRIBUTING TO THE CONVERGENCE OF ITS DEFINITION
}

\author{
Navonil Mustafee \\ University of Exeter Business School \\ University of Exeter \\ Exeter, EX4 4ST, UK \\ Anatoli Djanatliev
Computer Networks \& Communication Systems
University of Erlangen-Nuremberg
D-91058 Erlangen, GERMANY

\author{
Sally Brailsford \\ Southampton Business School \\ University of Southampton \\ Southampton, SO17 1BJ, UK
}

Tillal Eldabi

Brunel Business School

Brunel University London

Uxbridge, UB8 3PH, UK

Martin Kunc

Warwick Business School

University of Warwick

Scarman Rd

Coventry, CV4 7AL, UK

\author{
Andreas Tolk \\ The MITRE Corporation \\ Modeling, Simulation, Experimentation, \\ \& Analytics \\ Hampton, VA, 23666, USA
}

\begin{abstract}
There is a growing trend in the number of M\&S studies that report on the use of Hybrid Simulation. However, the meaning and the usage of the term varies considerably. Indeed, the hybrid simulation panel during last year's conference (WSC2016) laid bare the strong views, from the panelists and audience alike, as to what constitutes a hybrid model and what is new? The ensuing debate set the scene for this year's paper, in which we discuss the various perspectives on hybrid simulation by focusing on three aspects: its definition, its purpose and its benefits. We hope this paper will pave the way for further studies on this subject, with the objective of achieving a convergence of the definition of hybrid simulation.
\end{abstract}

\section{INTRODUCTION}

The Hybrid Simulation track at the WSC has continued to grow over the last four years. This is representative of the growing interest in this topic, and indeed, reflects the volume of publications accessible through scholarly outlets. However, the meaning and the usage of this term varies considerably, as the hybrid methodology is not precisely defined (Balaban, Hester, and Diallo 2014). Most of the studies refer to a pair-wise (e.g., Heath et al. 2011) or full combination (e.g., Djanatliev and German 2013) of the three most commonly applied simulation techniques, namely the Discrete-Event Simulation (DES), System Dynamics (SD), and Agent-Based Simulation (ABS). Furthermore, new terms have been introduced which, arguably, have the same meaning, e.g., multi-method simulation, multiparadigm modeling, cross-paradigm simulation, mixed-modeling and combined simulation. From the literature, we find numerous examples of "hybrid" models for specific use-cases. However, what makes those models "hybrid"? Is this only the application of two or more simulation paradigms? Why is hybridization necessary or useful in a certain case? What are the major steps to develop a hybrid model? 
Are there synergies to be derived through hybrid simulation or is it just a passing fad? These are only but a sub-set of the questions that make it difficult for new researchers to better appreciate this combined modelling approach; such questions affords even experienced researchers and groups only a restricted view on the matter. Conceptual papers and guidelines for hybrid simulation may help to define a structure, but there are only a few of them. According to Eldabi et al. (2016), most of the hybrid attempts are "ad hoc and pragmatic with no clear methodology."

The paper presents the panelists' perspectives on hybrid simulation by focusing on three aspects: the definition, the purpose and the benefits of hybrid simulation. We hope that this work will lead towards achieving a convergence of the definition of hybrid simulation and clarify its purpose and benefits.

\section{THE CLASSIFICATION OF HYBRID SIMULATION IS IT'S DEFINITON (MUSTAFEE)}

The definition of hybrid simulation will need to align with the historic, albeit infrequent, use of the term (see Case 1 below), it should be representative of its present use (Case 2) and, ideally, put in place an intellectual scaffolding to support future research (Case 3 and 4).

Alignment with the Past (Case 1): In Shantikumar and Sargent (1983), the authors provide a unified definition of the combined use of analytical models (defined by them as a set of equations that characterize a system/problem entity) with dynamic simulation models. They refer to this as "Hybrid Simulation/Analytic Models and Modeling". The definition of hybrid simulation should therefore recognize the historic use of the term and what this meant.

Alignment with Present Practice (Case 2): In academic discourse, much of the recent discussions around hybrid simulation has focused on the combined use of DES/SD/ABS. With DES/ABS being discrete-time and SD continuous-time, the definition has gradually gravitated towards the mixed application of simulations developed using the two modelling methods. Thus, DES-SD, ABS -SD, and ABS-DES-SD, all qualify as hybrid simulation. Most researchers agree that the uptake of model hybridization has increased with the availability of simulation packages which aid the development of such mixed models in a single modelling environment (refer to the views of other panelists).

Alignment with Future M\&S Research (Case 3): DES, SD and ABS are only a subset of available simulation techniques; other approaches include Monte Carlo Simulation (MCS), mesoscopic modelling which places itself between continuous and discrete models (Reggelin and Tolujew 2011), simulation of Petri Nets, Computational Fluid Dynamics (CFD), etc. Taking the case of Monte Carlo, there are studies on the combined use of MCS with DES for quantifying supply chain disruption risk (Schmitt and Singh 2009), use of ABS and MCS for solving real-world newsvendor problem (Negahban 2013), combined application of ABS, Petri Nets and MCS in assessing risks related to runway incursion (Stroeve, Blom and Bakker 2013), etc. However, these are often ignored in discussion pertaining to Case 2. Is an artificial boundary being drawn around techniques which could qualify to be the building blocks of hybrid simulation? Are definitions being shaped by the software tools available and the approaches they support? The definition of hybrid simulation should not be restricted to particular techniques but instead, allow for the exploration of synergies that could be achieved through assessment of the wider range of M\&S approaches. Future research should not be restricted only to methods commonly used in operations management and allied subjects, but instead investigate M\&S approaches used in disciplines such as engineering, finance and health economics.

Alignment with Broader Range of Operational Research (OR) Techniques (Case 4): Case 1 presents Shantikumar and Sargent's (1983) definition of hybrid simulation/analytical model, which was on the combined use of $M \& S$ with mathematical programming (equations). However, there exist numerous studies that have complemented M\&S approaches with a wider array of OR techniques, with the objective of enhancing specific stages of a simulation study. For example, the use of Soft Systems Methodology (SSM) in the conceptual modelling stage of a simulation study (Kotiadis, Tako, and Vasilakis 2014), combined application of Game-theoretic Modelling with simulation approaches for 
behavior validation - refer to Mustafee in panel paper by Eldabi et al. (2016), using Forecasting with DES (Harper and Mustafee 2017).

In laying the basis of what I term as hybrid simulation, I revisit Mingers and Brocklesby's (1997) definition of paradigm, methodology, technique and tool, and adapt it for hybrid study. This resonates the authors' view that the terms are defined and used mainly for consistency, and, "it must be recognized that these are not claimed to be 'correct' in some sense, and that inevitably some latitude will be required in applying them across a variety of domains" (Mingers and Brocklesby 1997).

- Paradigm: I distinguish between qualitative (interpretive, subjective, soft - ibid) and quantitative (positivist, objective, hard) paradigms; I place $M \& S$ in the quantitative paradigm. If qualitative approaches are used, e.g., in conceptual modelling phase, then it is an example of Multi-Paradigm Hybrid Study. As will be seen later, Hybrid Systems Modelling (Eldabi et al. 2016) encompasses such studies.

- Methodology: Methodologies generally develop within a paradigm and usually embody its philosophical assumptions (ibid). In a quantitative paradigm I distinguish between discrete and continuous methodologies. A Multi-Methodology Hybrid Study is one which has both Discrete and Continuous elements, e.g., SD-DES, SD-ABS.

- Technique: Techniques exist within the context of methodologies and have well defined purposes, e.g., DES (ibid). I distinguish between techniques such as DES (event list/queuing theory) and ABS (time stepped/emergence) under discrete methodology, and SD (stock and flow) and CFD (numerical approach) under continuous methodology. A Multi-Technique Hybrid Study is one which uses two or more techniques under the same methodology, e.g., using CFD to model traffic flow (Sun 2011) with SD to investigate strategic policy related to transportation at urban level (Shepherd 2014). If follows that, a Multi-Methodology, Multi-Technique Hybrid Model is one which uses a combination of techniques from both discrete and continuous methodologies, with at least two techniques from either of the two techniques. Studies demonstrating the combined application of SD-DES-ABS is an example of this.

- Tool: I define these as M\&S packages which can be used to "perform a particular technique" (ibid), and more recently, can execute multiple techniques that are classified under one or more methodologies. Discussion of the tool is not important for the purposes of my classification scheme.

My definition takes into account, (a) the alignment with four cases identified as being important in the context of hybrid research and practice, and (b) the definitions of paradigm, methodology and techniques. However, instead of trying to converge on an all-encompassing meaning of the term, I propose a Classification Scheme that represents Four Types of Hybrid Simulation, with each Type having a functional definition. A classification scheme also has the benefit of being extensible, thus allowing the accommodation of new types of hybrid models that may be realized in the future. The four types of hybrid models (Type A, B, C and D) that I propose are (Figure 1):

- Type A - Multi-Methodology Hybrid Simulation - Models of these type align with present practice (Case 2). There are numerous studies that have used SD-DES and SD-ABS.

- Type B - Multi-Technique Hybrid Simulation - Although these align with present practice (Case 2, e.g., use of ABS-DES models), there is some debate as to whether these could be called as hybrid since both techniques conform to discrete methodologies. In my classification, a combined application of ABS-DES is Type B hybrid simulation since there are fundamental differences in the execution of the simulation logic, and which makes them agreeable to model particular category of problems (top-down queuing approach versus bottom-up emergence). 
- Type C - Multi-Methodology, Multi-Technique Hybrid Simulation - This aligns with Case 2 (present practice, e.g., ABS-DES-SD models) and also accommodates future hybrid studies that is specific to M\&S (Case 3).

- Type D - Hybrid Systems Model, includes Multi-Paradigm Hybrid Systems Model - Type D is Hybrid Systems Model/Modelling (HSM), which is defined as the combined application of simulation with wider Operations Research (OR) techniques that can be applied to one or more stages of a simulation study - this was previously referred to as Hybrid M\&S Study by Powell and Mustafee (2016). This aligns with Case 1 and encompasses Shantikumar and Sargent's (1983) original use of hybrid simulation/analytical model and the four defined Classes of such models. An example of HSM is the combined application of mathematical modelling/optimization approaches with simulation models, e.g., use of load plan heuristics with ABS (Mustafee and Bischoff 2016) this is Class I hybrid simulation/analytical model according to Shantikumar and Sargent (1983). Further, Type D also aligns with Case 4 - the combined use of OR techniques with M\&S approaches. When Soft OR techniques are used with M\&S, e.g., SSM-DES (Kotiadis, Tako, and Vasilakis 2014) and QSD-DES (Powell and Mustafee 2016), then we have a special case of Type D model with intersecting paradigms. I refer to this as Type D.1 or Multi-Paradigm Hybrid Model. Type D and D.1 are referred to as hybrid model (rather than hybrid simulation) since only one constituent of the combined model is a simulation model; the other can be a method/technique from either Soft (qualitative) or Hard (quantitative) OR and not necessarily related to model execution.



Figure 1: Classification of Hybrid Simulation (Types A-D) with examples.

The purpose and benefits of hybrid simulation will be dependent on the type of model being implemented. The benefits accrued through Type D/D.1 models can relate to several stages of a M\&S study, unlike Types A-C where the purpose and benefits are mostly to do with model execution.

\section{THE HOLY GRAIL REVISITED (BRAILSFORD)}

\subsection{What is the Definition?}

On the face of it, my personal definition is very simple: hybrid simulation is one single conceptual simulation model that, when implemented in computer software, uses more than one simulation paradigm. Of course when you try to unpick this a little, it is not quite so simple. What exactly is one single conceptual model? And what is a simulation paradigm?

When I first got interested in hybrid modeling, at the turn of the century (Brailsford et al. 2003), my answer to the second question at least was straightforward: DES and SD. At the time these two approaches seemed totally distinct and complementary. Naively, I thought combining them in one single conceptual model would be relatively easy, and the real challenge would lie in the technical aspects of implementing this model in computer software. DES and SD used totally different software tools, and at 
the time I was not aware of any software that enabled the modeler to use both simultaneously. The DES and SD modeling communities were separate: they had their own journals and conferences and it was rare to see SD papers presented at WSC. Since then, of course, things have moved on. Tools like AnyLogic (The AnyLogic Company n.d.) have become mainstream and the boundaries between specialist DES and SD software tools have become blurred, with each trying to incorporate aspects of the other. Moreover, a third paradigm, agent-based modeling (ABM), has moved out of the predominantly research domain into the practical world of the industrial engineer.

As I became more and more interested in the topic, I realized that the first question was actually the more complex of the two. While many other researchers had considered the issue of combining methods generally (Mingers 2001; Munro and Mingers 2002) or DES and SD in particular (Chahal and Eldabi 2008), it was a paper by Morecroft and Robinson (2005) that really opened my eyes to the fact that DES and SD modelers simply saw the world differently, and would conceptualize the same problem in totally different ways. This was far more complex and subtle than merely developing models in which some variables were continuous and others discrete, and led to a series of research projects (e.g. Viana et al. 2014) and WSC papers (Brailsford et al. 2010; Brailsford et al. 2013; Viana 2014) which involved the search for the "holy grail" of a truly integrated philosophical approach. I still believe this exists, but I don't think we have reached it yet.

\subsection{What is the Purpose?}

The reason for my original interest in hybrid simulation was that I believed, and still do, that most realworld problems which are tackled with simulation cannot be solved by DES or SD alone but need a combination of the two. This was particularly the case in my main research field, healthcare, where "everything affects everything else" and variability and uncertainty are key features. The original problem area in my 2003 paper, Emergency Room (ER) modeling, is a case in point: nearly all previous ER models in the literature used DES only, but omitted many crucial interactions and feedbacks between the ER and the rest of the hospital, not to mention the world outside the hospital from where patients come! Moreover, I now believe that ABM has opened up the possibility of modeling other problem features that DES and SD, either alone or in combination, cannot capture.

Of course, the same thinking underpins mixed methodologies in general, and none of this is new. What are all models for? Solving problems ... making better decisions ... generating insights ... achieving understanding between diverse stakeholders ... the list goes on. People have used mixed modeling methodologies for decades (Jackson and Keys 1984) and it is rare in practice that even a supposedly "single method" simulation project does not include some kind of problem structuring method as a precursor to developing the actual model.

I end with a confession. While I am mainly motivated by the need to create better models to tackle real-world problems, I have to say that sometimes, mere curiosity has also been a factor.

\subsection{What are the Benefits?}

For me, the benefits are inextricably linked with the purpose. The benefit of choosing any modeling approach (or combination of approaches) is to enable the model to achieve its purpose more effectively, more quickly, more easily, and more cheaply.

- More effectively? I strongly believe that in the long run, hybrid approaches will lead to more useful models that better represent the real-world problem and provide better solutions. Viana (2014) compares developing a hybrid model in AnyLogic with a "composite" model where the DES and SD components were written in specialist DES and SD software - Simul8 (Simul8 Corporation n.d.) and Vensim (Ventana Systems Inc. n.d.), and argues that an experienced user of these single-paradigm 
tools would probably still prefer to use them. However, he has been exclusively using AnyLogic for the past two years and is now something of a convert.

- More quickly? Currently, in my experience, the opposite is true ... both in terms of model development time and runtime. However, I am sure this will change in future.

- More easily? This is unclear. AnyLogic is still the only genuinely multi-paradigm tool and is definitely easier to use than it was ten years ago. Nevertheless, it still requires some familiarity with Java.

- More cheaply? Interestingly, AnyLogic now offers a free version for students: the same strategy used in the early days of Simul8. Maybe a new generation of students will grow up using AnyLogic and then ask their future employers to buy it...

To conclude, back in 1977 there was a WSC paper (Standridge et al. 1977) which described a hybrid continuous/discrete model of the primary health care system in Indiana. Hybrid simulation is clearly not quite such a new idea as we thought when we started the Hybrid mini-track in WSC'14, even though hybrid modeling software like AnyLogic was obviously not available in 1977.

\section{MORE THAN JUST COMBINING SIMULATION PARADIGMS (DJANATLIEV)}

\subsection{What is the Definition?}

Recently, the lack of a consistent term usage for hybrid simulation has been identified and discussed by numerous authors. In Mustafee et al. (2015) Padilla mentions the need for an agreed consensus and a formal definition. The author uses the term hybrid simulation within the context of combined continuous and discrete simulation. Moreover, in the last year's panel paper (Eldabi et al. 2016) Sargent refers to hybrid simulation as "a term with multiple meanings and usages." Looking back to historical lessons, the author discusses the distinction between hybrid simulation and hybrid models. Thereafter, hybrid models allow to solve different mathematical models using a digital computer whereas hybrid simulation is related to a method solving problems using a computer "having analog and digital capabilities."

Following these examples, we can derive that hybrid simulation should contain continuous and discrete components, or to be more general, components having a different resolution for time progress. However, there are many publications where this term is used differently, mainly triggered by powerful software packages. Such tools allow to develop models by using many different features without a formal distinction of applied modeling techniques (e.g., AnyLogic). Typical examples are models where DES components and ABS components are combined to solve a problem. Both simulation methods are related to each other and their common basis for time progress is discrete.

However, do we really need a differentiation therefore? Wouldn't it be better to use the term hybrid simulation for all kinds of models with combined simulation paradigms without a distinction between continuous and discrete? It is a fact that combining DES with SD is not the same as coupling DES with ABS. Usually, there are different purposes to develop the one or the other combined model, and we apply different methods to connect continuous with discrete model parts. That are important reasons why I argue that two different terms and definitions are useful. In the following, I use the term hybrid simulation as presented in the above definition. When referring to a more general combination of any kinds of simulation paradigms without a distinction between continuous and discrete, I suggest to use the term multi-paradigm modeling/simulation, as the corresponding tools already do (i.e., Anylogic).

Coming back to the title of this section, I further argue that hybrid simulation is more than just a combination of simulation paradigms. We need an agreed understanding and clearly defined modeling steps which are collected in common guidelines. Those guidelines shall also include a set of examples and extracted best practices from specific use-cases. 


\subsection{What is the Purpose?}

Many problems that shall be solved by simulation can be modeled by one particular modeling technique. For example, continuous and flow-based structures can be represented by SD which is based on differential equations (e.g., energy or money flows). DES and ABS models are well-suited for behavioral models and microscopic coherences (e.g., individual decisions). However, there are problems that refer to both; continuous and discrete structures at the same time. In general, it is probably possible to use one simulation technique to represent such problems as well, but such a modeling process can be compared to "a case of hammering in a screw" (Brailsford, Desai, and Viana 2008).

Consequently, the main purpose of hybrid simulation is to give the modeler a broader flexibility to apply problem-oriented modeling features. At the beginning of many simulation studies the models are usually small and can be easily developed by one simulation approach. However, when solving complex problems and considering different levels of abstraction in large models, a combination of continuous and discrete components is often required. For example, using hybrid methods, an energy system modeler may represent energy flows (e.g., energy consumption) by SD techniques, but discrete changes (e.g., switching lights on) affecting rates in SD models can be modeled by DES.

As already mentioned, we need guidelines and a collection of examples that help the model developer to represent such structures and to reuse existing techniques. An appropriate conceptual basis connecting SD and DES models can be found in the work of Chahal (2009). In Djanatliev and German (2015), we presented an approach to generate agents from SD models and five types of interaction between continuous and discrete model components.

\subsection{What are the Benefits?}

The benefits of hybrid simulation can be mainly derived from the previous subsection. The hybrid approach helps to flexibly combine existing models within a common large-scale context. Furthermore, it allows to use continuous and discrete techniques for solving complex problems. Hybrid simulation enables to start modeling by abstract high-level models using SD with low data requirements and to "zoom-in" certain model parts in order to represent more detailed structures by DES or ABS. This topdown approach avoids unnecessary data requirements in microscopic models.

In addition, hybrid simulation can be used to solve trade-offs between the accuracy of results and the simulation performance. In Djanatliev (2015), we have developed three models that represent the same problem in healthcare. The first model has been completely modeled using SD, the second model has been developed by ABS techniques. Thereafter, we have run them at the same computer. The SD model was finished after few seconds, even for a nationwide population size, but the ABS model was running for 1.5 hours and was not able to finish with more than 20,000 agents. However, due to a more detailed problem representation, the accuracy of the results was much better in ABS. In order to master this problem, we applied hybrid simulation techniques. In this case, we started by abstract SD models and "zoomed-in" important parts to represent them in a greater detail. Additionally, we have used "superagents" that represent similar individuals by one aggregated agent object. Finally, this third model resulted in an acceptable runtime and produced results comparable to the results of the ABS model.

\section{HYBRID SIMULATION: A LIFECYCLE VIEW (ELDABI)}

\subsection{What is the Definition?}

The main purpose of using simulation is to solve complex problems that cannot be solved analytically or on a back of an envelope. Consequently, there are many attempts that have been given different terms. The term hybrid simulation has generated more interest in the past decade due to the increasing complexity of systems (Zulkepli and Eldabi 2015). This gave rise to a very important philosophical, yet quite operational, question: what is Hybrid Simulation? 
There is a number of frameworks that describe simulation approaches and their steps. For simplicity and for the purpose of this panel - we will assume that a simulation modeling study encompasses 3 phases: (1) Conceptual Modeling, (2) Simulation Modeling, and (3) Analytical Modeling. Phase 1 is mainly about problems structuring and conceptual modeling phase. Phase 2 is where the simulation takes place. This will be based on data and computer models. Phase 3 takes the outcomes of Phase 2 for further analysis and recommendations. It is commonly known that these phases are not strictly sequential, i.e. there may be loops back from Phase 2 to Phase 1 and from Phase 3 to Phase 2. The main focus of the hybridization will be during Phase 2 "Simulation".

The term Hybrid Simulation can be applied for any synchronous or asynchronous deployment of two or more simulation approaches within the simulation phase. To this end the following terms could be retermed as Hybrid simulation if they are used in this phase: (1) Multi- or Cross- Paradigm can be termed has Hybrid when two or more paradigms are employed during the simulation phase either in parallel or sequentially. Multi- or Combined Simulation is also the use of two or more simulation approaches and running them sequentially or in parallel. The most important elements in a Hybrid Simulation is the two or more simulations that are running during the simulation phase in parallel or sequentially.

\subsection{What is the Purpose and What are its Benefits?}

It is widely reported that Hybrid Simulation allows modelers and problem owners to gain better understandings of complex systems as it allows modelers to assess the problems from different dimensions (Zulkepli and Eldabi 2015). In the ever rising complexity of the modern world and the abundance of information outlets, Hybrid Simulation is becoming an important field within the Modeling and Simulation arena. There are many hybrid models developed and used in various areas such as in manufacturing, software development, supply chain, construction and healthcare (Martin and Raffo 2000; Rabelo et al. 2003; Helal et al. 2007; Venkateswaran et al. 2006; Lee at al. 2007; Chahal et al. 2009). All of these agree on the fact that Hybrid Simulation ought to be applied to solve complex problems associated with convoluted systems and where a single simulation approach may not be able to capture all the relevant details.

\section{HYBRID MODELS: BEYOND SIMULATION (KUNC)}

\subsection{What is the Definition?}

It is important to make a distinction between modelling and simulation. One of the fundamental reasons for making this distinction is that modelling is hybrid by nature since the model conceptualization stage, which is a very important stage in DES and SD, can be performed using any methodology that is not necessarily related with the simulation method, e.g. value chain or cognitive mapping. The conceptualization stage defines the objectives, system level representation (boundaries), inputs, outputs, content, assumptions and simplifications. However, the model development, e.g. equations and data, is constrained by the type of simulation method selected together with model simulation. Model simulation, as the process of execution that takes the model through state changes over time (Borschev and Filippov 2004), was also constrained by the type of simulation method employed until the development of multiparadigm simulation tools based on software engineering concepts, such as object-oriented languages (ibid). Therefore, the focus on the definition of hybrid simulation is on the second part of the modelling process: model development and simulation.

To put into context, hybridization is not a practice unique to the field of simulation. Operational Research/Management Science field has a long tradition on multi-methodologies and mixing methods (Morgan, Howick and Belton 2017). The key discussion in this literature is the existence of philosophical, methodological and technical considerations in mixing methods (Morgan et al. 2017). The reason for mixing methods is that real-world problems are usually very complex so they require different methods to 
address the multiplicity of dimensions of a problem. Additionally, all methods have different strengths and weaknesses so mixing methods can overcome the limitations of one method. Munro and Mingers (2002) found that mixing methods is usually a result from requirements and ad hoc/emergence situations rather than thorough thinking. Moreover, mixing methods that are incommensurables can be detrimental (Mingers 2011). In terms of mixing methods, there are many possibilities but my focus is on enrichment and integration for this definition. Enrichment occurs when the use of one method or elements of it intends to add value to another method so there is no emergence of something different from the existing methods (Morgan et al. 2017). Integration happens when the elements of both methods are integrated, so their original paradigms are abandoned, in a way to generate a new method.

Therefore, my definition of hybrid simulation is "the process of model development and simulation that uses more than one simulation method in order to enrich or integrate them depending on the purpose of the model."

\subsection{What is the Purpose?}

The purpose of hybrid simulation is intrinsically related with the conceptualization of the model. For example, does the model need to address issues at different levels of aggregation? Then, an ABM may be useful to combine with a SD model. However, the key question is what is offering the ABM that cannot be handled by the SD if it is developed carefully and vice versa. Additionally, why is necessary to have a model with different levels of aggregation? Rahmandad and Sterman (2008) provided an in-depth comparison of the insights generated by two separate SD and ABM models when both models are parameterized similarly. The results obtained did not differ significantly in key metrics. They concluded that detail and flexibility of ABM are useful when there is a clear understanding of the agents and they have characteristics that preclude the homogenization of their behaviours and there are high chances of random events without knowing their specific impacts (Rahamandad and Sterman 2008). But SD is useful when there is a need for fast models without knowing how the processes happen at micro-levels. Therefore, a hybrid model dealing with issues of aggregation needs to be carefully thought in terms of integration into a unique new method or just enriching either SD or ABM, e.g. such as in the case of Anylogic models.

Other issues that may originate from the conceptual model are the realization of subsystems working with different time (continuous vs discrete), different time horizons (short vs. long), or there is no adequate knowledge of how system states change over time. When the system modelled has some of these conditions, it is clear that elements of SD model can enrich DES models, such as the paper authored by Viana et al. (2014).

Finally, an issue originated from the model conceptualization process is the boundary of the model. Broader boundaries require incorporating feedback processes endogenously so SD models can enrich DES or ABM models (Rahamandad and Sterman 2008).

From a brief analysis of model purpose, I would say that most of the hybrid models are related to enrichment of a core simulation method with elements of other methods. Morgan et al. (2017) found evidence of only one hybrid SD/DES fully integrated so further exploration is necessary.

\subsection{What are the Benefits?}

The benefits of hybrid simulation are multiple. First, if there is a need to achieve a complete representation of the real world problem (after the model conceptualisation process) that is not possible with only one simulation method, a hybrid model will provide more accuracy in the problem representation and solution. Second, hybrid models can help to uncover problems when only one simulation method is employed. Third, hybrid models may integrate more stakeholders into the implementation of model solutions since hybrid models can address multi-level issues. 
Mustafee, Brailsford, Djanatliev, Eldabi, Kunc, and Tolk

\section{THE NEED FOR CONCEPTUAL ALIGNMENT (TOLK)}

This following section is focusing on the need for conceptual alignment of all participating systems, phases, and solutions that are contributing to a hybrid modeling and simulation approach.

\subsection{What is the Definition?}

The currently most including definition for hybrid modeling and simulation was given by Powell and Mustafee (2016). We differentiate between hybrid M\&S studies, in which various modeling paradigms are applied in an orchestrated set of simulation tools, and hybrid M\&S systems, where several such paradigms are used within one simulation. These are obviously not mutual exclusive, as a hybrid M\&S study can utilize one or several hybrid M\&S systems.

But how to define hybrid? In all definitions - biologically and technically - a hybrid is the result of merging two or more components of different categories to generate something new, that combines the characteristics of these components into something more useful. A mule is a biological hybrid, the crossbred of a donkey and a horse with better endurance and a longer useful lifespan than its parents. Crops grown from hybrid seeds produce plants of high quantity and quality. A hybrid car combines the advantages of gasoline engines and electric motors. Hybrid golf clubs combine the characteristics of wood and iron. Hybrids take two - or more - components and create something better. What does this means for simulation?

Fishwick (2007) addresses modeling paradigms regarding modeling methodologies - such as discrete event systems, SD, and agent based approaches - and model types - such as ordinary differential equations, process algebra, and temporal logic. Yilmaz et al. (2007) introduced the ideas of supporting multiple resolutions, stages, and even multiple models, although they did not use the term hybrid, but multisimulation in their work. Many other terms were introduced that address challenges of hybrid M\&S, many of them compiled by Lynch and Diallo (2015). Simulation can furthermore differ in scale, scope and structure, and they can support different concepts of time (Goldstein and Khan 2017). A consistent definition requires to define a taxonomy of terms that capture the various M\&S categories from which the simulation components can be selected from. A hybrid M\&S uses therefore two or more components of different $\mathrm{M} \& \mathrm{~S}$ categories to implement a common conceptual model, namely the one that is constructed to support the underlying $M \& S$ effort. This results in several requirements to be fulfilled by hybrid M\&S solutions, namely:

- In order to contribute to a common objective, hybrid M\&S must share some common properties, their behavior, and relations needed to reach the objective.

- Hybrid M\&S uses two or more components of different M\&S categories that are logically consistent regarding the common propertied concepts, their behavior, and their relations they are supporting.

- The data used to drive the hybrid M\&S solutions are part of the components of different M\&S categories, i.e., the underlie the same constraints.

I summarized recent work on interoperability and composability in Taylor et al. (2015) and proposed that the definition composability is the consistent representation of truth in all participating systems. As such, the final requirement needed for the definition of hybrid M\&S is that the components of different M\&S categories must be composable, i.e., may not lead to different truth values depending on which components is used to answer a certain question of interest within the underlying M\&S effort that is supported by the composition. The resulting definition is therefore as follows:

Hybrid $M \& S$ results from using two or more components of different $M \& S$ categories to generate something new, that combines the characteristics of these components into something more useful for the underlying $M \& S$ effort to be supported that are composable under the constraints of this effort. 


\subsection{What is the Purpose?}

The components of different M\&S categories utilized by the hybrid M\&S effort must be logically equal, but they are not the same. Each component highlights a different facet of the underlying solution. Hester and Tolk (2010) show a traffic example to address the benefits of applying methods of the full M\&S spectrum to address complex problems, which is a hybrid M\&S study approach:

- SD helps to identify bottlenecks within the traffic flow of a broader region, such as the Hampton Roads area in South-East Virginia, providing a macro-level view of traffic.

- These bottlenecks are then further evaluated in more detail using DES approaches of a higher resolution. The results are the identification critical traffic joints, such as crossings, bridges, tunnels, etc., which build the mesa-level of the traffic system.

- The use of high resolution ABMs allows now the modeling and evaluation of induvial behavior on the traffic flow, the micro-level of the system of interest.

Djanatliev and German (2015) show by generalizing this idea that the purpose of this kind of hybrid M\&S is to cover the different aspects of behavior on the macro-, meso-, and micro-level of systems. The first purpose is to cover various abstraction levels.

However, it is often beneficial to use different modeling approaches on the same abstraction level as well, as each modeling approach has its strengths and weaknesses. Within the military application domain, the use of architecture frameworks is often mandatory for system development. Among others, Mittal (2006) showed how the architecture artifacts can be used to model the system behavior, and Garcia and Tolk (2015) embedded such system simulations into a context for operational and portfolio analyses. The different models allow to emphasize different aspects and facets of the system. The second purpose is to cover various aspects and facets of systems on the same abstraction level.

Over the lifetime of a system or a task, the questions, the abstraction level of interest, and the aspects on each of these levels change. While during development a highly detailed view of all system components is required, once the system runs as desired a higher level view is often used for operation and maintenance, requiring different models. The third purpose is to cover all lifecycle phases of a simulation supported effort.

Finally, different communities use different models to capture their knowledge, often focusing on methods that are close to domain-specific languages. Complex problems are rarely solvable by one of these communities alone, so that increasingly multi-, inter-, and transdisciplinarity approaches are needed that allows the experts from the various discipline to utilize those models and methods they are most comfortable with contributing to common research. The fourth purpose is to enable and support multi-, inter-, and transdisciplinarity simulation-based research, including the applicability of domain-specific languages, methods, and models.

\subsection{What are the Benefits?}

Interestingly enough, the first volume of the IBM Systems Journal featured an article about the use of simulation for systems engineering (Smith 1962). The benefits of combining various modeling paradigms is also featured in the simulation community since its early days, examples are Conway, Johnson, and Maxwell (1959) and the follow up article by Conway (1963), or Zeigler and Ören (1986).

Although an integrated hybrid M\&S approach is the desired objective, the realities tend to drive the solution to community-driven implementations. This will likely be the case for some time to come. We will continue to see domain-specific solutions, support of specific life-cycles of interest to the community, and focus on particular facets of the overall challenge. Researchers will continue to rely on systems with which they are most comfortable: their own! 
The benefits of hybrid M\&S is to combine two or more composable approaches enabling consistent $M \& S$ support addressing all aspects and facets on all abstraction levels over the whole life cycle, supporting the domain-specific needs of the supporting researchers.

\section{CONCLUSION}

The Hybrid Simulation Track is currently in its fourth year (2014-2017), but its inception could be traced back to WSC2012 (Berlin) and WSC2013 (Washington) where the focus was on the "Combined Application of Operations Research (OR)/Simulation Techniques." Initially, the idea was to introduce a track which focused mainly on the methodological aspects of combining classical OR methods (not limited to analytical methods and optimization) with $M \& S$ techniques. The ensuing debate resulted in naming the track as hybrid simulation as it was thought to be a better fit for an M\&S conference. This also took into account the growing volume of papers that were on the combined use of DES/SD/ABS. The track has, however, continued to advocate and seek original contributions that capitalize on research being done by two distinct communities (OR and M\&S) and beyond, but with an intersecting interest on the use of scientific methods to help make better decisions. As we debate the definition, purpose and benefits of hybrid simulation, we must not lose sight of the pivotal position of the track in encouraging M\&S researchers to come out of their traditional comfort zones in terms of methodologies and techniques and advocate the need to promote collaboration with researchers from other fields. For the hybrid simulation track this is only the start!

\section{REFERENCES}

Balaban, M., P. Hester, and S. Diallo. 2014. "Towards a Theory of Multi-method M\&S Approach: Part I'. In Proceedings of the 2014 Winter Simulation Conference, edited by A. Tolk, S. D. Diallo, I. O. Ryzhov, L. Yilmaz, S. Buckley, and J. A. Miller, 1652-1663. Piscataway, New Jersey: Institute of Electrical and Electronics Engineers, Inc.

Brailsford, S. C., L. C. Churilov, and S. K. Liew. 2003. "Treating Ailing Emergency Departments with Simulation: An Integrated Perspective". In Health Sciences Simulation, edited by J. Anderson and E. Katz, 25-30. San Diego, USA: Society for Modeling and Computer Simulation.

Brailsford S. C., M. S. Desai, and J. Viana. 2010. "Towards the Holy Grail: Combining System Dynamics and Discrete Event Simulation in Healthcare". In Proceedings of the 2010 Winter Simulation Conference. B. Johansson, S. Jain, J. Montoya-Torres, J. Hugan, and E. Yücesan, 2293-2303. Piscataway, New Jersey: Institute of Electrical and Electronics Engineers, Inc.

Brailsford S. C., J. Viana, S. P. Rossiter and A. R. Channon. 2013. "Hybrid Simulation for Health and Social Care: The Way Forward, or More Trouble than it's Worth?" In Proceedings of the 2013 Winter Simulation Conference, edited by R. Pasupathy, S.-H. Kim, A. Tolk, R. Hill, and M. E. Kuhl, 258269. Piscataway, New Jersey: Institute of Electrical and Electronics Engineers, Inc.

Chahal, K., and T. Eldabi. 2008. "Applicability of Hybrid Simulation to Different Modes of Governance in UK Healthcare". In Proceedings of the 2008 Winter Simulation Conference, edited by S. J. Mason, R. R. Hill, L. Mönch, O. Rose, T. Jefferson, J. W. Fowler, 1478-1483. Piscataway, New Jersey: Institute of Electrical and Electronics Engineers, Inc.

Chahal, K. 2009. "A Generic Framework for Hybrid Simulation in Healthcare." Ph.D. thesis. Brunel Business School, Brunel University London.

Conway, R. W. 1963. "Some Tactical Problems in Digital Simulation.” Management Science 10:47-61.

Conway, R. W., B. M. Johnson, and W. L. Maxwell. 1959. "Some Problems of Digital Systems Simulation." Management Science 6(1):92-110.

Djanatliev, A. 2015. "Hybrid Simulation for Prospective Healthcare Decision-Support: System Dynamics, Discrete-Event and Agent-Based Simulation”. Ph.D. thesis. Dr. Hut. Universität Erlangen-Nürnberg. Germany. 
Djanatliev, A., and R. German. 2013. "Prospective Healthcare Decision-Making by Combined System Dynamics, Discrete-Event and Agent-Based Simulation". In Proceedings of the 2013 Winter Simulation Conference, edited by R. Pasupathy, S.-H. Kim, A. Tolk, R. Hill, and M. Kuhl, 270-281. Piscataway, New Jersey: Institute of Electrical and Electronics Engineers, Inc.

Djanatliev, A., and R. German. 2015. "Towards a Guide to Domain-specific Hybrid Simulation". In Proceedings of the 2015 Winter Simulation Conference, edited by L. Yilmaz, W. K. V. Chan, I. Moon, T. M. K. Roeder, C. Macal, and M. D. Rossetti, 1609-1620. Piscataway, New Jersey: Institute of Electrical and Electronics Engineers, Inc.

Eldabi, T., M. Balaban, S. Brailsford, N. Mustafee, R. E. Nance, B. S. Onggo, and R. G. Sargent. 2016. "Hybrid Simulation: Historical Lessons, Present Challenges and Futures". In Proc. of the 2016 Winter Simulation Conference, edited by T.M. Roeder, P.I. Frazier, R. Szechtman, et al., 1388-1403. Piscataway, New Jersey: Institute of Electrical and Electronics Engineers, Inc.

Fishwick, P. A. (Ed.). 2007. Handbook of Dynamic System Modeling. Boca Raton, Florida: Chapman \& Hall/CRC Press, Taylor and Francis Group.

Garcia, J. J., and A. Tolk. 2015. "Executable Architectures in Executable Context Enabling Fit-forpurpose and Portfolio Assessment". Journal of Defense Modeling and Simulation 12(2):91-107.

Goldstein, R. and A. Khan. 2017. "A Taxonomy of Event Time Representations". In Proceedings of the Spring Simulation Multiconference, 1037-1048. San Diego, CA: Society of Modeling and Simulation.

Harper, A., and Mustafee, N. 2017. "A Hybrid Approach Using Forecasting and Discrete-Event Simulation for Endoscopy Services." In Proceedings of the 2017 Winter Simulation Conference, edited by W. K. V. Chan, A. D'Ambrogio, G. Zacharewicz, N. Mustafee, G. Wainer, and E. Page (to appear). Piscataway, New Jersey: Institute of Electrical and Electronics Engineers, Inc.

Heath, S. K., S. C. Brailsford, A. Buss, and C. M. Macal. 2011. "Cross-Paradigm Simulation Modeling: Challenges and Successes". In Proceedings of the 2011 Winter Simulation Conference, edited by S. Jain, R. R. Creasey, J. Himmelspach, K. P. White, and M. Fu, 2788-2802. Piscataway, New Jersey: Institute of Electrical and Electronics Engineers, Inc.

Hester, P. T., and A. Tolk. 2010. "Applying Methods of the M\&S Spectrum for Complex Systems Engineering". In Proceedings of the Spring Simulation Multiconference, 75-82. San Diego, CA: Society for Computer Simulation International.

Jackson, M. C., and Keys, P. 1984. "Towards a System of Systems Methodologies". Journal of the Operational Research Society 35(6):473-486.

Kotiadis, K., A. Tako, and C. Vasilakis. 2014. "A Participative and Facilitative Conceptual Modelling Framework for Discrete Event Simulation Studies in Healthcare." Journal of the Operational Research Society 65(2):197-213.

Lee, S. H., S. Han, and F. Peña-Mora. 2007. "Hybrid System Dynamics and Discrete Event Simulation for Construction Management". In Proceedings of the 2007 ASCE International Workshop on Computing in Civil Engineering, edited by L. Soibelman and B. Akinci, 232-239. Reston, Virginia.

Lynch, C. J., and S. Y. Diallo. 2015. "A Taxonomy for Classifying Terminologies that Describe Simulations with Multiple Models". In Proceedings of the 2015 Winter Simulation Conference, edited by L. Yilmaz, W. K. V. Chan, I. Moon, T. M. K. Roeder, C. Macal, and M. D. Rossetti, 1621-1632. Piscataway, New Jersey: Institute of Electrical and Electronics Engineers, Inc.

Martin, R. H. and D. Raffo. 2000. "A Model of the Software Development Process using both Continuous and Discrete Models". Software Process: Improvement and Practice 5(2-3):147-157.

Mingers, J. 2001. "Combining IS Research Methods: Towards a Pluralist Methodology". Information Systems Research 12(3):240-259.

Mingers, J. 2011. "Clarification or Confusion: Response to Harwood". Journal of the Operational Research Society 62(4):809-81.

Mingers, J., and J. Brocklesby. 1997. Multimethodology: Towards a Framework for Mixing Methodologies". Omega 25(5):489-509. 
Mittal, S. 2006. "Extending DoDAF to Allow Integrated DEVS-based Modeling and Simulation". Journal of Defense Modeling and Simulation 3(2):95-123.

Morecroft, J., and S. Robinson. 2005. "Explaining Puzzling Dynamics: Comparing the Use of System Dynamics and Discrete-Event Simulation". In 23rd International Conference of the System Dynamics Society, 17-21.

Morgan, J. S., S. Howick., and V. Belton. 2017. "A Toolkit of Designs for Mixing Discrete Event Simulation and System Dynamics". European Journal of Operational Research 257(3):907-918.

Munro, I., and J. Mingers, J. 2002. "The Use of Multimethodology in Practice-Results of a Survey of Practitioners". Journal of the Operational Research Society 53(4):369-378.

Mustafee, N., and E. E. Bischoff. 2013. "Analysing Trade-offs in Container Loading: Combining Load Plan Construction Heuristics with Agent-based Simulation." International Transactions in Operational Research 20(4):471-491.

Mustafee, N., J. H. Powell, S. C. Brailsford, S. Diallo, J. Padilla and A. Tolk. 2015. "Hybrid Simulation Studies and Hybrid Simulation Systems: Definitions, Challenges, and Benefits". In Proceedings of the 2015 Winter Simulation Conference, edited by L. Yilmaz, W. K. V. Chan, I. Moon, T. M. K. Roeder, C. Macal, and M. D. Rossetti, 1678-1692. Piscataway, New Jersey: Institute of Electrical and Electronics Engineers, Inc.

Negahban, A. 2013. "A Hybrid Simulation Framework For the Newsvendor Problem with Advertising and Viral Marketing." In Proceedings of the 2013 Winter Simulation Conference, edited by R. Pasupathy, S.-H. Kim, A. Tolk, R. Hill, and M. E. Kuhl, 1613-1624.Piscataway, New Jersey: Institute of Electrical and Electronics Engineers, Inc.

Powell, J. H., and N. Mustafee. 2016. "Widening Requirements Capture with Soft Methods: An Investigation of Hybrid M\&S Studies in Healthcare." Journal of the Operational Research Society (online first). http://rdcu.be/n4ov.

Rabelo, L., M. Helal, Y. J. Son, A. Jones, J. Min, and A. Deshmukh. 2003. "New Manufacturing Modeling Methodology: A Hybrid Approach to Manufacturing Enterprise Simulation." In Proc. of the 2003 Winter Simulation Conference, edited by S. Chick, P. J. Sánchez, D. Ferrin, and D. J. Morrice, 1125-1133. Piscataway, New Jersey: Institute of Electrical and Electronics Engineers, Inc.

Rahmandad, H., and Sterman, J., 2008. "Heterogeneity and Network Structure in the Dynamics of Diffusion: Comparing Agent-based and Differential Equation Models". Management Science 54(5):998-1014.

Reggelin, T., and T. Juri. 2011. "A Mesoscopic Approach to Modeling And Simulation of Logistics Processes". In Proceedings of the 2011 Winter Simulation Conference, edited by S. Jain, R. R. Creasey, J. Himmelspach, K. P. White, and M. Fu, 1508-1518.Piscataway, New Jersey: Institute of Electrical and Electronics Engineers, Inc.

Schmitt, A. J., and M. Singh. 2009. "Quantifying Supply Chain Disruption Risk using Monte Carlo and Discrete-Event Simulation". In Proceedings of the 2009 Winter Simulation Conference, edited by M. D. Rossetti, R. R. Hill, B. Johansson, A. Dunkin, and R. G. Ingalls, 1237-1248. Piscataway, New Jersey: Institute of Electrical and Electronics Engineers, Inc.

Simul8 Corporation. Simul8 - http://www. simul8.com. Accessed 17.08.17.

Smith, E.C. Jr. (1962). "Simulation in Systems Engineering”. IBM Systems Journal 1(1):33-50.

Shanthikumar, J. G., and R. G. Sargent. 1983. "A Unifying View of Hybrid Simulation/Analytic Models and Modeling". Operations Research 31(6):1030-1052.

Shepherd, S. P. “A Review of System Dynamics Models Applied in Transportation”. Transportmetrica B: Transport Dynamics 2(2):83-105.

Standridge, C., C. Macal, A. B. Pritsker, H. Delcher, and R. Murray. 1977. "A Simulation Model of the Primary Health Care System in Indiana". In Proceedings of the 1977 Winter Simulation Conference, 349-358. Piscataway, New Jersey: Institute of Electrical and Electronics Engineers, Inc. 
Stroeve, S. H., H. A. P. Blom, and G. J. Bakker. 2013. "Contrasting Safety Assessments of a Runway Incursion Scenario: Event Sequence Analysis Versus Multi-agent Dynamic Risk Modelling." Reliability Engineering \& System Safety 109:133-149.

Sun, D. 2011. "In-depth Analysis of Traffic Congestion using Computational Fluid Dynamics (CFD) Modelling Method." Journal of Modern Transportation 19(1):58-67.

Taylor, S. J. E., A. Khan, K. L. Morse, A. Tolk, L. Yilmaz, J. Zander, and P. J. Mosterman. 2015. "Grand Challenges for Modeling and Simulation: Simulation Everywhere - From Cyberinfrastructure to Clouds to Citizens". Simulation 91(7):648-665.

The AnyLogic Company. AnyLogic - https://www.anylogic.com/. Accessed 17.08.17.

Venkateswaran, J., Y. J. Son, A. T. Jones, and H. S. J. Min. 2006. "A Hybrid Simulation Approach to Planning in A VMI Supply Chain”. International Journal of Simulation \& Process Mod. 2:133-149.

Ventana Systems Inc. Vensim - www.vensim.com. Accessed 17.08.17.

Viana J., S. C. Brailsford, V. Harindra, and P. R. Harper. 2014. "Combining Discrete-Event Simulation and System Dynamics in a Healthcare Setting: A Composite Model for Chlamydia Infection". European Journal of Operational Research 237(1):196-206.

Viana J. 2014. "Reflections on Two Approaches to Hybrid Simulation in Healthcare". Proceedings of the 2014 Winter Simulation Conference, edited by A. Tolk, S. Y. Diallo, I. O. Ryzhov, L. Yilmaz, S. Buckley, and J. A. Miller, 1585-1596. Piscataway, New Jersey: IEEE.

Yilmaz, L., A. Lim, S. Bowen, S., and T. Oren. 2007. "Requirements and Design Principles for Multisimulation with Multiresolution, Multistage Multimodels". In Proceedings of the 2007 Winter Simulation Conference, edited by S. G. Henderson, B. Biller, M.-H. Hsieh, J. Shortle, J. D. Tew, and R. R. Barton, 823-832. Piscataway, New Jersey: Institute of Electrical and Electronics Engineers, Inc.

Zeigler, B. P., and T. I. Ören. 1986. "Multifacetted, Multiparadigm Modelling Perspectives: Tools for the 90's". In Proc. of the 1986 Winter Simulation Conference, edited by J. Wilson, J. Henriksen, and S. Roberts, 708-712. Piscataway, New Jersey: Institute of Electrical and Electronics Engineers, Inc.

Zulkepli, J., and T. Eldabi. 2015. "Towards a Framework for Conceptual Model Hybridization in Healthcare". In Proceedings of the 2015 Winter Simulation Conference, edited by L. Yilmaz, H. K. Chan, I. C. Moon, T. Roeder, C. M. Macal, and M. D. Rossetti, 1597-1608. Piscataway, New Jersey: Institute of Electrical and Electronics Engineers, Inc.

\section{AUTHOR BIOGRAPHIES}

NAVONIL MUSTAFEE is Senior Lecturer at UOE Business School, UK.n.mustafee@exeter.ac.uk.

SALLY BRAILSFORD is Professor of Management Science at University of Southampton, UK. s.c.brailsford@soton.ac.uk.

ANATOLI DJANATLIEV is Assistant Professor at University of Erlangen-Nuremberg, Germany. anatoli.djanatliev@fau.de.

TILLAL ELDABI is a Senior Lecturer at Brunel Business School, UK. tillal.eldabi@brunel.ac.uk.

MARTIN H KUNC is an Associate Professor of Management Science at Warwick Business School, University of Warwick. martin.kunc@wbs.ac.uk.

ANDREAS TOLK is Technology Integrator for the Modeling, Simulation, Experimentation, and Analytics Division of The MITRE Corp., Hampton VA USA. atolk@mitre.org. 